\title{
Myostatin and Sarcopenia: Opportunities and Challenges - A Mini-Review
}

\author{
Thomas A. White ${ }^{a}$ Nathan K. LeBrasseur ${ }^{a-c}$ \\ ${ }^{a}$ Robert and Arlene Kogod Center on Aging, ${ }^{b}$ Department of Physical Medicine and Rehabilitation, and \\ 'Department of Physiology and Biomedical Engineering, Mayo Clinic, Rochester, Minn., USA
}

\section{Key Words}

Aging $\cdot$ Frailty $\cdot$ Skeletal muscle $\cdot$ Atrophy $\cdot$ Growth and differentiation factor · Drug discovery · Obesity · Diabetes

\begin{abstract}
The progressive loss of skeletal muscle mass, strength and/ or function with advancing age, termed sarcopenia, poses a major threat to independence and quality of life. Therefore, there is significant merit in better understanding the biology of sarcopenia and developing therapeutic interventions to prevent, slow or reverse its progression. Since the discovery of myostatin, a potent negative regulator of growth that is highly enriched in skeletal muscle, there has been great interest in it as a potential mediator of sarcopenia as well as a therapeutic target. The complex biology of myostatin, the promise of myostatin inhibition as an effective means to counter sarcopenia, and the challenges facing its clinical translation are reviewed herein.

(c) 2014 S. Karger AG, Basel
\end{abstract}

\section{Introduction}

Humans achieve peak skeletal muscle mass in mid-life and then experience a progressive decline that can equal $50 \%$ by the ninth decade. The significant loss of skeletal muscle mass, strength and/or function with advancing age is termed sarcopenia. Sarcopenia has dire conse-

\section{KARGER}

E-Mail karger@karger.com

www.karger.com/ger quences for skeletal muscle performance, physical function and metabolism in older persons, and thus, poses a major medical and economic threat to society. The biological mechanisms that underlie sarcopenia remain incompletely understood, but are influenced by multiple factors including age-associated changes in hormones, sex steroids, physical inactivity, and comorbid conditions such as heart failure, cancer, and diabetes. As a result, untangling the causal molecular pathways in attempt to identify therapeutic targets to prevent, delay or reverse sarcopenia has proven challenging. In recent years there has been significant interest in growth and differentiation factor-8 (GDF-8), or myostatin, which functions as a powerful negative regulator of muscle growth [1]. The purpose of this review is to highlight the opportunities for myostatin inhibition as a strategy to counter sarcopenia, as well as discuss the challenges that have confronted its progression.

\section{Myostatin Signaling in Skeletal Muscle}

Myostatin is a highly conserved member of the transforming growth factor- $\beta$ superfamily. It is abundant in skeletal muscle, but also expressed to a lesser extent in adipose tissue and cardiac muscle [1,2]. Myostatin signals through the activin type IIB receptor (ActRIIB), which is expressed ubiquitously and forms a heterodimer with activin-like kinase 4 (ALK4) or ALK5. The intracel- 
lular serine/threonine kinase domain of ALK4 and 5 phosphorylates Smad2 and 3, which form a complex with Smad4. This complex translocates to the nucleus to regulate the transcription of genes involved in the proliferation and differentiation of skeletal muscle precursor cells $[3,4]$ and protein degradation pathways in mature myofibers [5]. The activation of Smad2 and 3 by myostatin also inhibits the Akt/mammalian target of rapamycin (mTOR) pathway in response to pro-growth signals (e.g. insulin and IGF-1), and therefore, suppresses protein synthesis [6]. As a result of its actions on cell growth and differentiation and intracellular catabolic and anabolic signaling pathways, myostatin strongly affects skeletal muscle development and postnatal growth. Indeed, deletion and loss of function mutations in myostatin cause skeletal muscle hyperplasia (an increase in the number of skeletal muscle fibers) and hypertrophy (an increase in the size of skeletal muscle fibers). These cellular adaptations yield a hypermuscular phenotype in multiple species, including humans [7]. Therefore, myostatin inhibition provides a promising means to attenuate or reverse skeletal muscle loss in the context of sarcopenia as well as cachexia (disease-associated muscle loss), and to enhance skeletal muscle regeneration in the context of congenital disease (i.e. muscular dystrophies) and injury.

\section{Myostatin in Aging and Disease}

The influence of advancing age on the abundance and/ or activity of myostatin is not yet clear. An early crosssectional study of younger, middle-aged and older men and women suggested that serum myostatin levels increase with advancing age, are highest in 'physically frail' older women, and are inversely associated with skeletal muscle mass [8]. Yet several subsequent reports have failed to show age-related differences in either circulating myostatin-immunoreactive protein or skeletal muscle myostatin mRNA levels [e.g. 9, 10]. These disparate findings suggest myostatin may not be a primary driver of sarcopenia, or may instead highlight the complexities related to myostatin and its measurement. First, myostatin abundance may not reflect myostatin activity. Indeed, myostatin is generated as a precursor protein that requires proteolytic cleavage to first remove its signal peptide and to then liberate an $\mathrm{N}$-terminal propeptide and a $\mathrm{C}$-terminal fragment. The mature biologically active form of myostatin is a disulfide-linked dimer of C-terminal fragments. Meanwhile, the N-terminal propeptide forms a complex with the mature C-terminal dimer and pre- vents its binding to ActRIIB [11]. The C-terminal dimer is liberated from the inactive, latent complex by the bone morpogenetic protein-1 (BMP-1)/tolloid family of metalloproteinases [12]. Despite improvements in the reliability and specificity of antibodies for myostatin compared to proteins with highly similar sequences (e.g. GDF-11), the methods used to quantify its abundance fail to distinguish between active and inactive, latent states. Whether or not their proportion may change as a function of age remains to be determined. Second, myostatin is further regulated by at least three interacting proteins, namely GDF-associated serum protein-1 (GASP-1), follistatin and follistatin-related gene (FLRG) (thoughtfully reviewed by Lee [13]). It is plausible that the abundance of these endogenous inhibitors of myostatin and/or the degree to which they interact with myostatin are independently affected by aging. However, a recent study reported that there were no significant differences in their abundance between younger healthy men and older men with either moderate or severe deficits in muscle strength [10]. The complex posttranslational regulation of myostatin activity has in part challenged efforts to explicitly define its role in sarcopenia. More advanced analytical techniques that can quantify the abundance of active relative to inactive myostatin, and its association with GASP-1, FLRG and follistatin, may help clarify this issue in a sizeable cohort of individuals in whom discriminating measures of skeletal muscle health have been conducted.

Of significant concern and relevance to the topic of sarcopenia is the prevalence of obesity in older adults. In the United States, for example, 35\% of persons over the age of 65 are obese [14]. In addition to elevating the risk for cardiovascular disease, type 2 diabetes, cancer, and other chronic conditions, obesity is a major cause of ageassociated functional limitations and disability [15]. Several lines of evidence suggest obesity may in part threaten skeletal muscle health through myostatin [reviewed in 16, 17]. For example, increased myostatin expression and secretion have been observed in skeletal muscle and adipose tissue samples derived from obese and extremely obese middle-aged women, in whom circulating concentrations of myostatin were found to be correlated with insulin resistance [18]. Conversely, the expression of myostatin in skeletal muscle appears to be significantly decreased in response to weight loss in obese patients undergoing gastric bypass surgery and is associated with improved insulin action $[19,20]$. In murine models of obesity and insulin resistance, pharmacological blockade of myostatin significantly improves muscle mass and multiple metabolic parameters, including glucose ho- 
meostasis, circulating triglycerides and circulating concentrations of the adipose tissue-derived cytokine, adiponectin $[21,22]$. These data support the premise that an increase in myostatin as a consequence of obesity compromises skeletal muscle health and systemic metabolism in a high proportion of older persons. Inhibiting myostatin may provide an effective means to simultaneously improve their physical function and metabolic health [23].

\section{Myostatin Inhibition as a Therapy for Sarcopenia}

Since its discovery, multiple strategies to disrupt myostatin activity have been developed, including neutralizing antibodies, propeptides, soluble ActRIIB receptors, and interacting proteins (GASP-1, follistatin and FLRG). Although alterations in myostatin expression and activity in the context of aging are incompletely understood, several of its characteristics make it a unique and desirable therapeutic target for sarcopenia. First, postnatal inhibition of myostatin unequivocally increases skeletal muscle mass in adult and older mammals [24, 25]. Specifically, we have observed that weekly injections of a neutralizing antibody to myostatin for 4 weeks significantly increases the relative weights of individual muscles by up to $17 \%$ in aged mice and improved indices of muscle performance and whole-body metabolism. In humans, administration of a single dose of soluble ActRIIB increased total body lean mass by $>1 \mathrm{~kg}$ in postmenopausal women [26]. Second, the effects of targeted inhibition of myostatin are highly specific to skeletal muscle. Despite profound increases in skeletal muscle in the various species in which myostatin has been mutated, the masses of other organs and prevalence of cancer appear largely unaffected. In fact, several lines of evidence suggest that disruption of myostatin signaling may positively influence age-associated changes in other tissues either directly or indirectly. Aged myostatin null mice exhibit increased bone mineral density and improved ejection fraction compared to wild-type mice [27]. Moreover, mice in which the myostatin gene has been mutated or deleted are resistant to diet-induced obesity, dyslipidemia, atherogenesis, hepatic steatosis, and macrophage infiltration/activation in adipose tissue and skeletal muscle $[28,29]$ (fig. 1 ). Third, myostatin is a very drugable protein given it is secreted, and therefore, accessible in the circulation. This characteristic has labeled myostatin as a myokine, which may partly explain how its deletion/inhibition impacts other tissues. Fourth, even partial reductions in myostatin abundance and/or activity are sufficient to drive meaningful changes in skeletal muscle mass and function. This can be inferred from studies using neutralizing antibodies that likely reduce total myostatin activity by a modest extent, as well as from whippets, a breed of racing dogs, that have a mutation in one copy of the myostatin gene and are more muscular and run significantly faster than wild-type peers [30]. Collectively, these traits underscore the promise of myostatin as a therapeutic target for sarcopenia.

Finally, it is critically important to note that all strategies to inhibit myostatin are not created equal. Neutralizing antibodies and propeptides are designed to specifically target myostatin, but other approaches are less discerning. For example, there is significant enthusiasm regarding the myostatin interacting protein, follistatin, as an anabolic intervention. This has partly resulted from the finding that transgenic muscle-specific overexpression of follistatin caused a further doubling, or in sum, a quadrupling of muscle mass in the double-muscled myostatin null mouse [31]. This suggests follistatin drives skeletal muscle growth in part through a mechanism other than inhibiting myostatin. However, if not confined to skeletal muscle, pharmacological administration of follistatin would modulate the activity of numerous molecules other than myostatin, including activin A, inhibin, follicle-stimulating hormone and several BMPs, and jeopardize pituitary and gonadal function. Similarly, pharmacological administration of soluble ActRIIB offers more horsepower with regard to muscle growth than more targeted means to inhibit myostatin but at the cost of less specificity and greater risk. Indeed, the abovementioned study of postmenopausal women whom received a single dose of soluble ActRIIB demonstrated marked suppression (43\%) of serum follicle-stimulating hormone and subsequent clinical trials in healthy volunteers and boys with Duchene's muscular dystrophy were terminated due to nose bleeds, gum bleeding and/or small dilated vessels within the skin [26]. These findings underscore the importance of determining what factors other than myostatin are modulated by interacting proteins and soluble ActRIIB that (1) drive muscle growth and, if specifically targeted, may potentially improve the efficacy of an intervention, and (2) negatively impact the health of other tissues and, if specifically avoided, would improve the overall safety of an intervention. Indeed, the tolerance for risk may differ depending on the indication and the treatment paradigm and its objectives. For example, a slightly higher risk may be acceptable for a short-term treatment in an older person with sarcopenia 


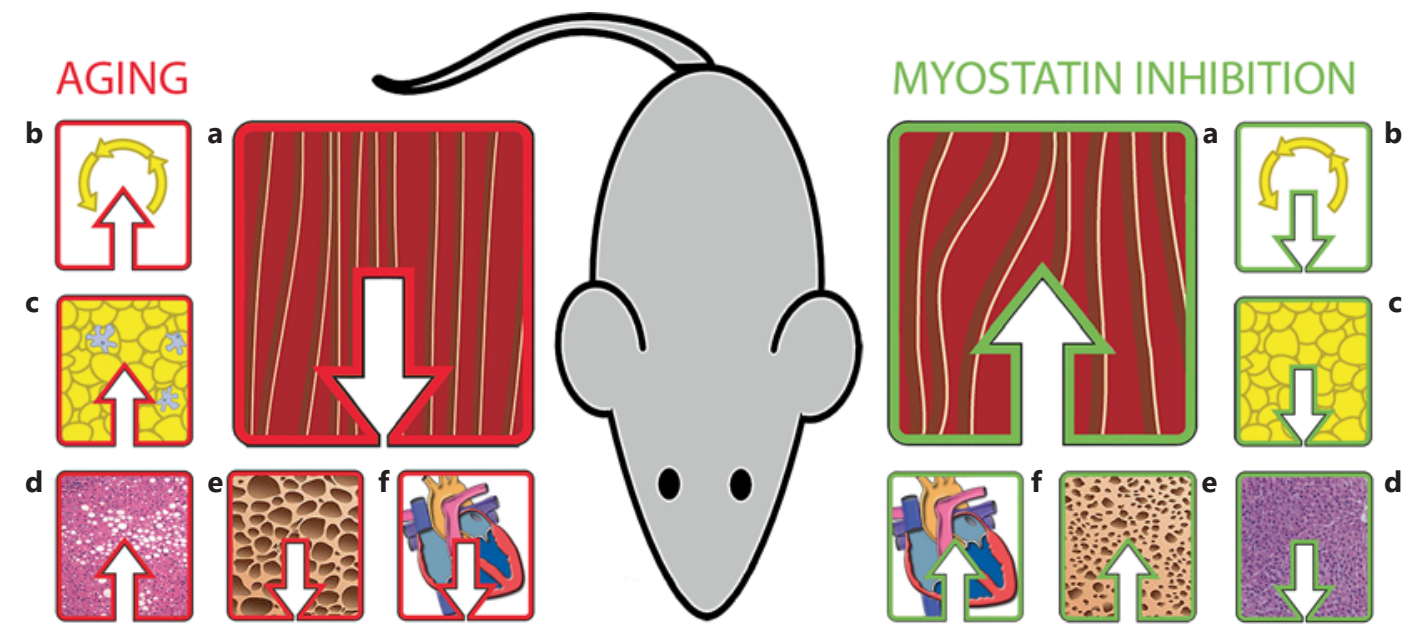

Fig. 1. Conditions of aging (left panel) affected by myostatin deletion and/or inhibition (right panel). a Age-associated reductions in muscle mass and fiber cross-sectional area are directly reversed by short-term myostatin inhibition $[25,26]$. b Increases in circulating glucose and triglyceride concentrations, elicited by high-fat feeding in mouse models and consequences of human aging, are significantly improved by myostatin inhibition and deletion [21, 22]. c, $\mathbf{d}$ Though the mechanisms appear to be indirect (e.g. as a result of greater metabolic demands of increased muscle mass), diet-mediated increases in fat mass, macrophage infiltration and inflammation (c) and ectopic lipid accumulation in the liver (d) are prevented in mice with mutations in the myostatin gene [28,

following hip fracture to prevent accelerated muscle loss and debilitation. In contrast, safety would be paramount for a long-term treatment intended for use in an older person with sarcopenia and slow gait speed to restore muscle mass and physical function. These considerations are not unique to myostatin inhibition, but relevant to ongoing discussions about interventions for sarcopenia.

\section{Conclusions}

Myostatin is a robust regulator of muscle development and postnatal growth whose activity is regulated by a number of complex posttranslational events. It is not clear whether or not the abundance or activity of myostatin is affected by aging or if myostatin plays a causal role in sarcopenia. Nonetheless, the inhibition of myostatin in adult and older animals significantly increases muscle mass and confers benefits upon measures of performance and metabolism. These effects along with the
32]. These observations could prove meaningful in the context of aging given the prevalence of obesity and type 2 diabetes. e Agerelated declines in bone mineral content and density are attenuated in myostatin-deficient mice [27]. Combined with its effects on muscle, myostatin inhibition could significantly impact osteoporotic fracture risk. Reductions in cardiac ejection fraction with advancing age are also prevented in myostatin-deficient mice [27]. f Moreover, myostatin deletion protects against aortic atheromatous lesions in mice genetically predisposed to atherosclerosis [29]. The mechanisms through which disruption of myostatin signaling conveys cardiovascular protection are not understood.

relative exclusivity of myostatin to muscle and the effects of its targeted inhibition on muscle make it a desirable and potentially safe and effective drug target for sarcopenia. Consequently, a number of strategies including antibodies, propeptides, interacting proteins and soluble decoy receptors are being investigated to inhibit its activity. While these approaches clearly increase muscle mass in the context of aging and disease, they differ in respect to their efficacy and safety. Ongoing research is needed to determine the optimal means to disrupt the activity of myostatin and related factors to safely increase muscle mass. This could lead to exciting and novel therapies for a growing number of older persons with sarcopenia and its disabling complications.

\section{Acknowledgements}

The authors would like to acknowledge the support of the Mayo Clinic Center for Cell Signaling in Gastroenterology (NIDDK P30DK084567), Mayo Clinic and a generous gift from Robert and Arlene Kogod. 


\section{References}

$>1$ McPherron AC, Lawler AM, Lee SJ: Regulation of skeletal muscle mass in mice by a new TGF- $\beta$ superfamily member. Nature 1997; 387:83-90.

-2 Sharma M, Kambadur R, Matthews KG, Somers WG, Devlin GP, Conaglen JV, Fowke PJ, Bass JJ: Myostatin, a transforming growth factor- $\beta$ superfamily member, is expressed in heart muscle and is upregulated in cardiomyocytes after infarct. J Cell Physiol 1999;180: $1-9$.

$>3$ Langley B, Thomas M, Bishop A, Sharma M, Gilmour S, Kambadur R: Myostatin inhibits myoblast differentiation by down-regulating MyoD expression. J Biol Chem 2002;277: 49831-49840.

4 Yang W, Zhang Y, Li Y, Wu Z, Zhu D: Myostatin induces cyclin D1 degradation to cause cell cycle arrest through a phosphatidylinositol 3-kinase/AKT/GSK-3 $\beta$ pathway and is antagonized by insulin-like growth factor 1 . J Biol Chem 2007;282:3799-3808.

5 Sartori R, Milan G, Patron M, Mammucari C, Blaauw B, Abraham R, Sandri M: Smad2 and 3 transcription factors control muscle mass in adulthood. Am J Physiol Cell Physiol 2009; 296:C1248-C1257.

6 Trendelenburg AU, Meyer A, Rohner D, Boyle J, Hatakeyama S, Glass DJ: Myostatin reduces Akt/TORC1/p70S6K signaling, inhibiting myoblast differentiation and myotube size. Am J Physiol Cell Physiol 2009; 296:C1258-C1270.

7 Schuelke M, Wagner KR, Stolz LE, Hubner C, Riebel T, Komen W, Braun T, Tobin JF, Lee SJ: Myostatin mutation associated with gross muscle hypertrophy in a child. N Engl J Med 2004;350:2682-2688

-8 Yarasheski KE, Bhasin S, Sinha-Hikim I, PakLoduca J, Gonzalez-Cadavid NF: Serum myostatin-immunoreactive protein is increased in 60 - to 92-year-old women and men with muscle wasting. J Nutr Health Aging 2002;6:343348.

$>9$ Welle S, Bhatt K, Shah B, Thornton C: Insulin-like growth factor-1 and myostatin MRNA expression in muscle: comparison between 62-77 and 21-31 year old men. Exp Gerontol 2002;37:833-839.

10 Ratkevicius A, Joyson A, Selmer I, Dhanani T, Grierson C, Tommasi AM, DeVries A, Rauchhaus P, Crowther D, Alesci S, Yaworsky P, Gilbert F, Redpath TW, Brady J, Fearon KC, Reid DM, Greig CA, Wackerhage H: Serum concentrations of myostatin and myostatininteracting proteins do not differ between young and sarcopenic elderly men. J Gerontol A Biol Sci Med Sci 2011;66:620-626.
11 Lee SJ, McPherron AC: Regulation of myostatin activity and muscle growth. Proc Nat Acad Sci USA 2001;98:9306-9311.

12 Wolfman NM, McPherron AC, Pappano WN, Davies MV, Song K, Tomkinson KN, Wright JF, Zhao L, Sebald SM, Greenspan DS, Lee SJ: Activation of latent myostatin by the BMP-1/tolloid family of metalloproteinases. Proc Natl Acad Sci USA 2003; 100:1584215846.

13 Lee SJ: Regulation of muscle mass by myostatin. Annu Rev Cell Dev Biol 2004;20:6186.

14 Fakhouri TH, Ogden CL, Carroll MD, Kit BK, Flegal KM: Prevalence of obesity among older adults in the United States, 2007-2010. NCHS Data Brief 2012, pp 1-8.

15 Seeman TE, Merkin SS, Crimmins EM, Karlamangla AS: Disability trends among older Americans: national health and nutrition examination surveys, 1988-1994 and 19992004. Am J Public Health 2010;100:100-107.

16 Allen DL, Hittel DS, McPherron AC: Expression and function of myostatin in obesity, diabetes, and exercise adaptation. Med Sci Sports Exerc 2011;43:1828-1835.

17 Lebrasseur NK: Building muscle, browning fat and preventing obesity by inhibiting myostatin. Diabetologia 2012;55:13-17.

-18 Hittel DS, Berggren JR, Shearer J, Boyle K, Houmard JA: Increased secretion and expression of myostatin in skeletal muscle from extremely obese women. Diabetes 2009;58:3038.

19 Milan G, Dalla Nora E, Pilon C, Pagano C, Granzotto M, Manco M, Mingrone G, Vettor $\mathrm{R}$ : Changes in muscle myostatin expression in obese subjects after weight loss. J Clin Endocrinol Metab 2004;89:2724-2727.

20 Park JJ, Berggren JR, Hulver MW, Houmard JA, Hoffman EP: GRB14, GPD1, and GDF8 as potential network collaborators in weight loss-induced improvements in insulin action in human skeletal muscle. Physiol Genomics 2006;27:114-121.

21 Akpan I, Goncalves MD, Dhir R, Yin X, Pistilli EE, Bogdanovich S, Khurana TS, Ucran J, Lachey J, Ahima RS: The effects of a soluble activin type IIB receptor on obesity and insulin sensitivity. Int J Obes (Lond) 2009;33: 1265-1273.
22 Bernardo BL, Wachtmann TS, Cosgrove PG, Kuhn M, Opsahl AC, Judkins KM, Freeman TB, Hadcock JR, LeBrasseur NK: Postnatal PPAR $\delta$ activation and myostatin inhibition exert distinct yet complimentary effects on the metabolic profile of obese insulin-resistant mice. PLoS One 2010;5:e11307.

23 LeBrasseur NK, Walsh K, Arany Z: Metabolic benefits of resistance training and fast glycolytic skeletal muscle. Am J Physiol Endocrinol Metab 2011;300:E3-E10.

24 Whittemore LA, Song K, Li X, Aghajanian J, Davies M, Girgenrath S, Hill JJ, Jalenak M, Kelley P, Knight A, Maylor R, O’Hara D, Pearson A, Quazi A, Ryerson S, Tan XY, Tomkinson KN, Veldman GM, Widom A, Wright JF, Wudyka S, Zhao L, Wolfman NM: Inhibition of myostatin in adult mice increases skeletal muscle mass and strength. Biochem Biophys Res Commun 2003;300:965-971.

25 LeBrasseur NK, Schelhorn TM, Bernardo BL, Cosgrove PG, Loria PM, Brown TA: Myostatin inhibition enhances the effects of exercise on performance and metabolic outcomes in aged mice. J Gerontol A Biol Sci Med Sci 2009;64:940-948.

26 Attie KM, Borgstein NG, Yang Y, Condon $\mathrm{CH}$, Wilson DM, Pearsall AE, Kumar R, Willins DA, Seehra JS, Sherman ML: A single ascending-dose study of muscle regulator ACE031 in healthy volunteers. Muscle Nerve 2013; 47:416-423.

27 Morissette MR, Stricker JC, Rosenberg MA, Buranasombati C, Levitan EB, Mittleman MA, Rosenzweig A: Effects of myostatin deletion in aging mice. Aging Cell 2009;8:573-583.

28 Wilkes JJ, Lloyd DJ, Gekakis N: Loss-of-function mutation in myostatin reduces tumor necrosis factor- $\alpha$ production and protects liver against obesity-induced insulin resistance. Diabetes 2009;58:1133-1143.

29 Tu P, Bhasin S, Hruz PW, Herbst KL, Castellani LW, Hua N, Hamilton JA, Guo W: Genetic disruption of myostatin reduces the development of proatherogenic dyslipidemia and atherogenic lesions in Ldlr null mice. Diabetes 2009;58:1739-1748.

30 Mosher DS, Quignon P, Bustamante CD, Sutter NB, Mellersh CS, Parker HG, Ostrander EA: A mutation in the myostatin gene increases muscle mass and enhances racing performance in heterozygote dogs. PLoS Genet 2007;3:e79.

$>31$ Lee SJ: Quadrupling muscle mass in mice by targeting TGF- $\beta$ signaling pathways. PLoS One 2007;2:e789.

32 McPherron AC, Lee SJ: Suppression of body fat accumulation in myostatin-deficient mice. J Clin Invest 2002;109:595-601. 